\title{
Fatty acids in focus - the good and the bad ones
}

I n 1993, trans fatty acids (TFA) came into focus as a possible factor contributing to diet-related disease risk. That turn in the fat quality debate was triggered mainly by new epidemiological data demonstrating a positive association between TFA intake and the risk of coronary heart disease (CHD). Intervention studies published in the early 1990 's showed that TFA not only increased the LDL-cholesterol level, but also decreased HDLcholesterol and increased lipoprotein (a). This led to similar conclusions in most nutrition recommendations seen during the last 10 years: TFA have effects that are at least as unfavourable as saturated fatty acids (SFA) and both should be reduced.

According to the Nordic nutrition recommendations 2004, SFA and TFA together should not exceed $10 \%$ of the energy (en $\%)$. The background of this recommendation is described in the article by Antii Aro, Jan I. Pedersen and Wulf Becker. Although a reduction of TFA as far as possible is recommended, emphasis is put on the reduction of SFA by about $1 / 3$ to achieve the recommended level of intake, which is estimated to reduce CHD risk by $30 \%$. Since the TFA intake in the Nordic countries has already decreased to $0.5-1.0 \mathrm{en} \%$, thanks to voluntary exchange of TFA for other fatty acids in most consumer products, it is estimated that a further corresponding decrease by 0.2-0.3 en $\%$ would have a small additional effect only.

In Denmark, the TFA story developed differently, as described in the article by Steen Stender, Jørn Dyerberg, and Arne Astrup. Already in 1994, the newly formed Danish Nutrition Council (DNC) issued a report recommending that the TFA content should not exceed $5 \%$ of the total fat in any food product. As in the other Nordic countries, the TFA intake fell substantially through voluntary actions by the food industry to replace TFA. Two new DNC reports in 2001 and 2003 concluded that the evidence that TFA is a risk factor for CHD had strengthened. Based on these reports, the Danish government introduced a law, effective from 2004, that the TFA content must not exceed $2 \%$ of total fat in any product. After 2 years, however, the European Commission asked Denmark to withdraw this law, which was thus not accepted on the EU level.

The main rationale behind the Danish TFA law was that, in spite of a favourable development in average TFA intake, as in the other Nordic countries, it was still possible in 2001 to compose "hightrans menus" with up to $30 \mathrm{~g}$ TFA from foods available in Denmark. After the legislation, this was reduced to a few grams. It is noteworthy, also for other nutrients, that a sizable number of consumers with excessively high (or low) intakes may be hidden behind average numbers.

Whereas recommendations to limit TFA intake relate mainly to the connection with CHD, the third TFA article in this issue, by Ulf Risérus, reviews the evidence of a relationship between TFA and diabetes. This review includes metabolic effects of one special TFA, conjugated linoleic acid (CLA), which are not as evidently positive as supposed in the marketing of CLA preparations.

Proceedings of the symposium "Novel aspects of fatty acids - nutrition and biological functions", Nafa2006, are published as a supplement to this issue of the Scandinavian Journal of Food and Nutrition. A summary of the symposium is included in this issue as well. We are obliged to the scientific and organising committee and the eminent speakers for this collection of state-of-the-art papers showing the many newly discovered effects of fatty acids, not least the long-chain polyunsaturated fatty acids, ensuring that fatty acids will remain in focus, in nutrition as well as in biomedicine in general.

Nils-Georg Asp

Editor-in-Chief

E-mail: asp@snf.ideon.se 